\title{
Tratamiento de aguas residuales mineras contaminadas con cobre mediante Lemna minor (lenteja de agua)
}

\section{Treatment of residual water contaminated with copper using Lemna minor (water bean)}

\author{
1.2Ávila Carhuallanqui, G.M.; ${ }^{2}$ Velit Villareal, C.; ${ }^{2}$ Ávila Pichiule, L.E; \\ ${ }^{2}$ Rosales Casas, S.; ${ }^{2}$ Manchego Chamorro, A.; ${ }^{3}$ Mora Velit, J. \\ Facultad de Ingeniería Química, Universidad Nacional del Centro del Perú \\ Email: gladyavi@hotmail.com
}

\section{Resumen}

El presente trabajo de investigación, tiene por objetivo evaluar la fitorremediación del ión cobre mediante la especie lenteja de agua (Lemna minor) en aguas residuales mineras; para ello, se preparó el agua con iones de cobre a una concentración de 4,780 ppm; teniendo como referencia la descarga de un efluente de aguas residuales mineras, el tejido vegetal estuvo varias semanas en contacto con el cobre, se preparó la solución nutritiva para la especie Lemna minor, se llevó a cabo el contacto de la planta con el agua con iones cobre y la solución nutritiva de la planta durante 5 semanas, cada semana se realizó el análisis de cobre en el agua, en la quinta semana la concentración de cobre en el agua fue de 1,502 ppm observándose una remoción de 68,57\% de cobre. Se concluye que la especie Lemna minor tiene capacidad depuradora del cobre en aguas residuales mineras.

Palabras Clave: fitorremediación, aguas residuales, tejido vegetal, solución nutritiva, ión de cobre

\begin{abstract}
The present research work has as objective evaluating the copper ion phytoremediation by means of the lentil water species (Lemna minor) in mining wastewater, for this the water with copper ions was prepared at a concentration of $4,780 \mathrm{ppm}$; having as reference the discharge of an effluent of mining wastewater, the vegetal tissue was in contact with copper for several weeks, then the nutrient solution for the Lemna minor species was prepared, the contact of the plant with the water was carried out with copper ions and the nutrient solution of the plant for 5 weeks, each week the copper in the water analysis was carried out, in the fifth week the copper concentration in the water was $1,502 \mathrm{ppm}$, having a copper removal of $68,57 \%$. This concludes that Lemna minor species has depurative capacity in mining wastewater.
\end{abstract}

Keywords: phytoremediation, wastewater, plant tissue, nutrient solution, copper ion 


\section{Introducción}

La problemática del agua, en estos tiempos, es álgido en todos los lugares del mundo, el Perú no es ajeno a ello, por ser un país minero requiere grandes cantidades de agua para las diversas etapas del proceso, estas aguas después del uso en este sector se transforman en aguas residuales con contenido de metales pesados, el cobre es uno de estos metales; es por ello que, en esta investigación se estudió su comportamiento frente a la planta acuática, denominada Lemna minor, la cual existe en la región.

Durante los últimos años, la contaminación en los cuerpos de agua ha ido aumentando a causa del mal manejo de las aguas residuales industriales y municipales. Por ello, es necesario desarrollar tecnologías que mejoren la calidad de los efluentes al más bajo costo, en este caso, la fitorremediación es una alternativa.

La fitorremediación, es una técnica de bajo costo que utiliza plantas para eliminar, transformar o estabilizar contaminantes que incluyen contaminantes orgánicos localizados en el agua, sedimentos o suelos. Las ventajas de la remediación fitosanitaria sobre la biorremediación inusual por microorganismos son que las plantas, como sistemas autotróficos con biomasa grande, requieren solo un aporte moderado de nutrientes y previenen la propagación de contaminantes a través de la humedad. Las plantas también proporcionan nutrientes para las bacterias de la rizosfera, lo que permite el crecimiento y el mantenimiento de una comunidad microbiana para una mayor desintoxicación contaminante (Cherian \& Margarida, 2005).

La fitorremediación usa plantas para limpiar ambientes contaminados. Las plantas pueden ayudar a limpiar muchos tipos de contaminantes, incluidos metales, pesticidas, explosivos y aceite. Sin embargo, funcionan mejor donde los niveles de contaminación son bajos porque las altas concentraciones pueden limitar el crecimiento de la planta y tomar demasiado tiempo para limpiar. Las plantas también ayudan a prevenir el viento, la lluvia y el flujo de agua subterránea de transportar contaminantes fuera del sitio a áreas circundantes o más profundo bajo tierra (A Citizen's Guide to Phytoremediation, 2012).

La fitorremediación se considera una tecnología respetuosa con el medio ambiente, que es una forma segura y, también, económica de eliminar contaminantes, en algunos casos haciendo el mismo trabajo que un grupo de ingenieros por una décima parte del costo. Sin embargo, dicha tecnología no puede ser necesariamente efectiva todo el tiempo o ser usado en todo tipo de sitios contaminados. Si la contaminación es demasiado profunda o si la concentración de compuestos tóxicos es demasiado alta, entonces las plantas solas no pueden remediar el suelo de manera eficiente (Cunningham, Berti \& Huang, 1995).

En este proceso, el mecanismo principal por el cual los metales se acumulan en la superficie de algunas plantas acuáticas, es cuando los iones del metal son secuestrados de la solución mediante distintos procesos como la quimiosorción, formación de complejos, intercambios iónicos, etc. (Stirk, 2001). Existen macrófitas acuáticas que tienen la habilidad para asimilar hasta cierto punto, todos los constituyentes del agua considerandos como contaminantes, estas se han empleado en la deteccion y remocion de sustancias en efluentes de aguas residuales domésticas e industriales (Martelo y Lara, 2012).

Hay muchos tipos diferentes de humedales, cada tipo específico tiene características únicas que lo identifican. Algunas áreas tienen diferentes tipos de humedales en un sitio, incluso se superponen. La identificación de humedales es una tarea muy importante en el mundo de hoy.

El objetivo de este estudio fue tratar las aguas residuales mineras con cobre mediante el uso de plantas hidrófilas como Lemna minor (lenteja de agua).

\section{Materiales y métodos}

\section{Preparación del agua residual sintética}

A partir de los datos recopilados, según el monitoreo de la Dirección General de Salud Ambiental - Digesa sobre el río Yauli, localizada en la provincia de Yauli del departamento de Junín, en el vertimiento de aguas residuales, se realizó la preparación del agua sintética conteniendo cobre a una concentración de 4,780 ppm.

\section{Recolección de la planta Lemna minor}

La recolección de la planta Lemna minor se hizo en el lugar de Pío Pata (Huancayo), específicamente de una pequeña laguna, donde se utilizó un colador para recoger la planta y un recipiente para guardarla, para luego ser llevado al Laboratorio de Bioprocesos de la Universidad Nacional del Centro del Perú.

\section{Procedimiento experimental}

Una vez preparada la muestra sintética de agua con iones cobre a una concentración de 4,780 ppm de solución con cobre para 47 L de solución, en el que incluye la solución nutritiva, se coloca la planta Lemna minor durante varias semanas, cada semana se llevó una muestra de agua al laboratorio de análisis de agua, para determinar la concentración de iones cobre en el agua, esto se hizo hasta que la concentración de cobre sea constante.

Este procedimiento se llevó a cabo en un humedal artificial, cuyas dimensiones fueron: altura: $21 \mathrm{~cm}$; ancho 1: 67 $\mathrm{cm}$; ancho 2: $31 \mathrm{~cm}$; largo 1: 85,5 cm; largo 2: 55,5 cm

La solución nutritiva estuvo compuesta por: KNO3 $(0,2$ g/L), Ca (NO3)2 (0,8 g/L), KHPO4 (0,2 g/L), MgSO4.7H2O $(0,2 \mathrm{~g} / \mathrm{L})$ y FePO4 $(0,1 \mathrm{~g} / \mathrm{L})$.

Según la descarga del río Yauli, se preparó una solución sintética de agua con iones cobre de concentración 4,780 ppm con el CuSO4.5H2O, para un humedal ar- 
tificial de capacidad 78,5 litros de volumen. El volumen de solución con iones cobre que se consideró, fue del 60 $\%$, equivalente a 47 litros.

Se colectó plantas de Lemna minor de su hábitat, la planta se adaptó en el humedal artificial, por 5 días, donde también se agregó la solución nutritiva luego de los 5 días se colectó la muestra de agua en un volumen de 10 $\mathrm{mL}$ cada 7 días, se analizó el cobre en las muestras de agua con el espectrofotómetro de UV-Vis.

\section{Resultados}

Según la Tabla 1, se observa que la concentración total de cobre en el agua en las muestras estudiadas varía de 3 432 a 1502 ppm.

Tabla 1

Concentración de cobre en el agua

\begin{tabular}{cc}
\hline Número de muestras & Cobre total (ppm) \\
\hline M-I & 3,432 \\
M-II & 2,865 \\
M-III & 1,885 \\
M-IV & 1,521 \\
M-V & 1,502 \\
\hline
\end{tabular}

La Tabla 2, muestra la concentración de cobre inicial en la muestra de agua residual antes del tratamiento.

Tabla 2

Concentración de cobre en la muestra inicial

\begin{tabular}{cc}
\hline Muestra inicial & Cobre total (ppm) \\
\hline M-i & 4,78 \\
\hline
\end{tabular}

Mayor remoción del cobre por L. minor se observa a los 28 días del tratamiento con $68,57 \%$.

Tabla 3

Remoción de cobre en el agua

\begin{tabular}{ccc}
\hline Muestra tratada & Cobre Total (ppm) & $\begin{array}{c}\text { Remoción de cobre } \\
\text { (\%) }\end{array}$ \\
\hline M-I & 3,432 & 28,20 \\
M-II & 2,865 & 43,82 \\
M-III & 1,885 & 60,56 \\
M-IV & 1,521 & 68,17 \\
M-V & 1,502 & 68,57 \\
\hline
\end{tabular}

\section{Discusión}

Con respecto al tiempo de absorción la eficiencia de remoción con mercurio lo determinó en 22 días (Arenas, 2011), en el presente estudio se determinó en 28 días o 4 semanas la remoción del ión cobre Se considera este tiempo porque entre la cuarta y quinta semana, se puede observar que la concentración de iones cobre son valores similares.

El porcentaje de remoción fue de 68,57\%, mientras que Jaramillo y Flores (2012), en la investigación con mercurio fue de 29,5\%; comparado con el presente estudio, se tiene mayor porcentaje de remoción del ión cobre, entonces la Lemna minor es una planta acuática apropiada para este tipo de fitorremediación. La comparación con la literatura se ha realizado con otros metales pesados.

\section{Conclusiones}

- La eficiencia de la fitorremediación del cobre en una solución acuosa con la especie lenteja de agua (Lemna minnor) se evaluó hasta un 68,57 \% de remoción.

\section{Referencias bibliográficas}

A Citizen's Guide to Phytoremediation. (2012). EPA,1.

Arenas Adolfo D. (2011). Evaluación de la planta Lemna minor como biorremediadora de aguas contaminadas con mercurio. Universidad Centro Occidental Lisandro Alvarado, Decanato de Agronomía, Dpto. De Química y Suelos, Cabudare, Estado Lara - Venezuela.

Arias, A., Alejandra, R., Fernández, V., \& Sánchez, N. E. (2016). Lenteja de agua (Lemna minor) para el tratamiento de las aguas residuales que provienen del lavado de la fibra de fique (Furcraea bedinghausii). Ingeniería y competitividad.

Arroyave, M. d. (2004). La lenteja de agua (Lemna Minor L.): Una planta acuática promisoria*. Revista EIA, 33-38.

Blowes, D. W. (2003). Mill tailings: hydrogeology and geochemistry. Environmental Aspects of Mine Wastes, 95-116.

Bres, P., D., C., Rizzo, P., \& La Rossa, R. (2012). Capacidad de las macrófitas Lemna minor y Eichhornia crassipes para eliminar níquel. Revista de Investigaciones Agropecuarias.

Ciencias (2000). Enciclopedias. España. Everest.

Chapman, B. M. (1983). Processes controlling metal ion attenuation in acid mine drainage streams. Geochimica et Cosmochimica Acta 47, 1957-1973.

Cherian, S., \& Margarida, O. M. (2005). Transgenic Plants in Phytoremediation: RecentAdvances and New Possibilities. Environmental Science \& Technology, 1.

Cunningham, S., Berti W. \& Huang J. (1995). Phytoremediation of contaminated soils. 
Culley, D.D., Rejma'nkova', E., Kvet, J., Frye, J.B., 2009. Production chemical quality and use of duckweeds (Lemnaceae) in aquaculture waste management and animal feeds. J. World Aquacult. Soc. 12, 27-49

Director General de la UNESCO. (1971). Ramsar Convention on Wetlands of International Importance especially as Waterfowl Habitat. Convention on Wetlands, (pág. 169). Iran.

EPA. (1994). Copper - Extraction and Beneficiation of Ores and Minerals. Washington, DC.

GBR. (2012). Mining in Turkey. Global Business Report. E\&MJ.

Go botany. (s.f.). Obtenido de https://gobotany. newenglandwild.org/species/lemna/minor/

Godfrey, R., Wooten, J., (1979). Aquatic and Wetland Plants of Southeastern United States, first ed. University of Georgia Press, Athens.

Gupta, C., Prakash, D., (2013). Duckweed: an effective tool for phyto-remediation. Toxicol. Environ. Chem. 95, 1256-1266.

Hernández, S., Fernández C. \& Baptista L. (2003). Metodología de la investigación.

Jaramillo, M., Flores E. (2012). Fitoremediación mediante el uso de dos especies vegetales Lemna minor (Lenteja de agua) y Eichornia crassipes (Jacinto de agua) en aguas residuales producto de la actividad minera. Cuenca. Ecuador.

Kutschera, U., Niklas, K., (2015). Darwin-Wallace Demons: survival of the fastest in populations of duckweeds and the evolutionary history of an enigmatic group of angiosperms. Plant Biol. 17, 24-32.

Martelo, J. y Lara Borrero, J. (2012). Macrófitas flotantes en el tratamiento de aguas residuales: una revisión del estado del arte. Ingeniería y Ciencia, 8(15): 221-243

Plants For A Future. (21 de Junio de 2009). Obtenido de https://www.pfaf.org/user/Plant.aspx? Latin Name $=$ Lemna + minor

Skillicorn, P., Journey, W., Spira, W., 1993. Duckweed Aquaculture, first ed. World Bank, Washington, DC.

Stirk, W., Van Staden J., (2001) Some Physical Factors Affecting Adsorption of Heavy Metals from Solution By Dried Brown Seaweed Material. South African Journal of Botany. 67. 615-619.

The Sierra Fund. (2008). Mining's Toxic Legacy: An Initiative to Address Mining Toxins in the Sierra Nevada.
Wetland Wildflowers of Illinois. (s.f.). Obtenido de http: / / www.illinoiswildflowers.info/wetland/ plants/cm_duckweed.htm

Yue, L. (2018). Interaction of $\mathrm{CuO}$ nanoparticles with duckweed (Lemna minor. L): Uptake, distribution and ROS production sites. Environmental Pollution, $1-30$.

Zimmo, O. (2003). Nitrogen Transformations and Removal Mechanisms in Algal and Duckweed Waste Stabilization Ponds (Ph.D. thesis). International Institute for Infrastructural Hydraulic and Environmental Engineering, Delft, Netherlands

Zhao, Z., Shi, H., Duan, D., Li, H., Lei, T., Wang, M., (2015a). The influence of duckweed species diversity on ecophysiological tolerance to copper exposure. Aquat. Toxicol. 164, 92-98.

Zhao, Y., Fang, Y., Jin, Y., Huang, J., Bao, S., Fu, T., (2015b). Pilot-scale comparison of four duckweed strains from different genera for potential application in nutrient recovery from wastewater and valuable biomass production. Plant Biol. 17, 82-90.

Zirschky, J., Reed, S., (1988). The use of duckweed for wastewater treatment. J. Water Pollut. Control Fed. 60, 1253-1258 